\title{
¿Cultivo de peces marinos? Hablemos de larvicultura en estanques
}

\author{
Sergio Escárcega Rodríguez
}

\section{Resumen}

En virtud de su vasto potencial biológico y adaptabilidad en agua marina, salobre y dulce, los eurihalinos - seres acuáticos capaces de vivir en un amplio rango de salinidad-podrían representar un nuevo rumbo en el cultivo de organismos acuáticos en México, con el desarrollo y expansión del cultivo de peces marinos tropicales, soportado en las especies eurihalinas de nuestros litorales.

Al momento, el cultivo de peces marinos en México se encuentra en etapa experimental, bajo esquemas de cultivo de alta tecnificación, con la producción en paralelo de alimento vivo y alcances productivos limitados. Aquí se presenta como alternativa un modelo de cultivo en estanques, que permite el manejo de grandes cantidades de larvas, con hasta $60 \%$ de sobrevivencia. La clave del éxito radica en acoplar la preparación de los estanques con el momento en que las larvas tienen dos días de nacidas, una opción que permitiría alcanzar la escala productiva necesaria para detonar nuevas cadenas de valor en sus distintos eslabones: producción primaria, procesamiento, distribución y comercialización, de alto impacto socioeconómico.

Palabras clave: piscicultura marina, larvicultura en estanques, manejo de la sucesión del zooplancton, producción masiva de crías de peces marinos, eurihalinos.

\section{LARVICULTURE OF MARINE FISH IN PONDS: AN AFFORDABLE OPTION TO MASSIFY FINGERLINGS PRODUCTION}

\begin{abstract}
By virtue of its vast biological potential and adaptability in marine, brackish and fresh water, euryhaline - aquatic organisms capable of living in a wide range of salinities-could mean a new direction in the farming of aquatic organisms in Mexico, by the development and growth of a tropical marine fish farming supported by the eurihaline species of our coastlines.

At the moment, larval cultivation with marine fish in Mexico is in an experimental stage, under hightech farming schemes, with parallel production of live food and limited productive reach. Here, we present a pond culture model as an alternative that allows the handling of large quantities of larvae, with survival margins that can reach up to 60\%. The key to success lies in the coupling of ponds preparation with the moment that two-day hatching larvae are available. An option that would allow to reach the necessary productive scale to detonate new value chains in their different links: primary production, processing, distribution and marketing.
\end{abstract}

Keywords: marine fish farming, larviculture in ponds, management of the succession of zooplankton, massif production of marine fish fingerlings, euryhaline organisms.

Dol: http://doi.org/10.22201/codeic.16076079e.2020.v21n2.a3 


\section{Sergio Escárcega Rodríguez}

Biólogo por la Facultad de Ciencias de la Universidad Nacional Autónoma de México (UnAm) y Maestro en Ciencias en Limnología y Acuicultura por el Instituto de Investigaciones sobre los Recursos Naturales de la Universidad Michoacana de San Nicolás de Hidalgo. Ha sido Jefe de los Centros Acuícolas de Tiacaque, México, y de El Peaje, San Luis Potosí, de la Secretaría de Pesca/ SEMARnAP, y Subdelegado de Pesca de la semarnap en los estados de Guanajuato y Michoacán, así como Subdirector de Fomento Pesquero y Acuícola de la Comisión de Pesca y Director de Contención del Deterioro Ambiental de la Secretaría de Urbanismo y Medio Ambiente del gobierno del estado de Michoacán de Ocampo.

Ha publicado artículos en materia de acuicultura y cambio climático, en memorias de congresos nacionales e internacionales y artículos en revistas indizadas y arbitradas; además de libros en materia de cultivo de peces. Ha sido comisionado por el gobierno de México en eventos de intercambio técnico y capacitación en materia acuícola en la República Popular China y en Puerto Montt, Chile. Actualmente se desempeña como consultor independiente. 


\section{Introducción}

La acuicultura parece un término extraño y alejado de nosotros, pero en realidad está presente en el salmón que comemos, en los pescados del supermercado, en las algas que adornan el sushi. Se trata de una práctica milenaria que cultiva organismos acuáticos como moluscos, peces, algas, pulpos, y que interviene en la crianza de estas especies para aumentar su producción. A la práctica que se dedica exclusivamente a los peces se le denomina piscicultura y suele dividirse de acuerdo con el tipo de agua donde residen los peces (marina, dulce o salobre, punto medio de salinidad entre ambas) y con la especie a la que pertenecen. Por tanto, la producción de larvas de peces, también llamada larvicultura, constituye una etapa clave para esta industria.

El tema de la seguridad alimentaria es un asunto de gran importancia. En la actualidad somos más de 7 mil millones de seres humanos en el planeta, aunque se espera que para la mitad de este siglo la cifra supere los 9 mil millones (Food and Agriculture Organization [FAO], 2016). La demanda de alimentos se incrementará, así como la presión sobre los recursos naturales, por lo que se deberán prever opciones para atender estas eventualidades; además de combatir la desnutrición, potenciar el desarrollo, reducir la pobreza y reforzar los sistemas alternativos de producción ambientalmente sostenibles.

México constituye una nación con una economía emergente, donde resulta necesario diversificar las opciones productivas para avanzar en la conservación del capital natural, la autosuficiencia alimentaria, la generación de empleos y el desarrollo regional. En este sentido, el potencial de crecimiento de la piscicultura marina es amplio si consideramos la disponibilidad de más de 11 mil kilómetros de litorales y la existencia de una fauna tropical diversa (Escárcega, 2005).

Un nuevo rumbo en la producción nacional acuícola podrá darse con el desarrollo de una piscicultura marina tropical soportada en las especies eurihalinas de peces de nuestros litorales, pues toleran amplias variaciones de salinidad en el agua, poseen un gran potencial biológico y versatilidad de cultivo.

Entre ellos, se destacan los robalos (Centropomidae) por sus altos índices de fecundidad (615 mil óvulos/kg), resistencia al manejo, tolerancia al hacinamiento y a condiciones cambiantes en la calidad del agua, rápido crecimiento (ÁlvarezLajonchére \& Tsuzuki, 2008) y el más elevado valor comercial de pescados y mariscos en México (conAPESCA, 2014). También, para ilustrar este aspecto, en la región del Pacífico Sur de México (Pacífico Transicional Mexicano) se perfilan, además de los robalos prieto (Centropomus nigrescens) y plateado (Centropomus viridis), otras especies con aptitud acuícola como el mero guasa (Epinephelus itajara), el guachinango (Lutjanus peru), el pámpano plateado (Trachinotus kennedyi) y la corvina blanca (Cynoscion albus) (Escárcega-Rodríguez, 2018). 
Figura 1. Momento culminante del desove en el sistema acoplado de desove e incubación (Escárcega, 2005).
Al manejar los procedimientos adecuados en la reproducción y crianza (ver figura 1) dichas cualidades permiten obtener crías a gran escala (decenas de millones por año) para los sistemas de engorda en sus distintos niveles (extensivo, semi-intensivo e intensivo) y para los sistemas de cultivo como estanques, jaulas, canales y encierros. En consecuencia, resulta posible consolidar nuevas cadenas de valor con especies que presentan un gran valor nutricional y una importante demanda en el mercado. Este es precisamente el propósito central del documento: dar a conocer una nueva forma de aumentar la producción actual de crías peces marinos a nivel comercial.

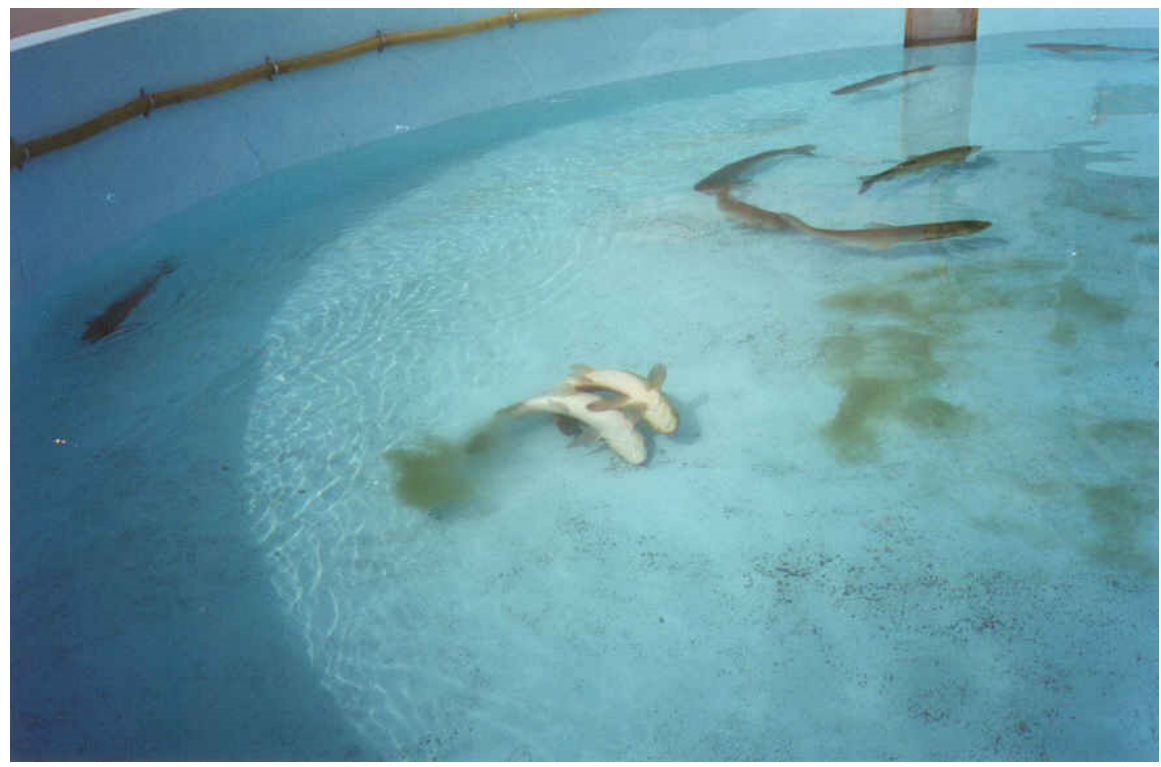

\section{Los retos para masificar la producción de crías}

La oportunidad de masificar la producción de larvas de peces marinos en estadio de alimentación - cuando han agotado sus reservas e inician su alimentación natural- exige el perfeccionamiento de tecnologías para la larvicultura y la crianza capaces de garantizar el abasto de crías y juveniles requeridos en los sistemas de engorda.

Al respecto, en México se han aplicado técnicas para la reproducción inducida y la larvicultura de robalos, pargos (Lutjanidae) y otras especies a nivel piloto-experimental. De esta manera, en la Unidad Académica Sisal-unAm, Yucatán, se ha realizado un importante trabajo con el robalo blanco del Atlántico (Centropomus undecimalis), la corvina pinta (Cynoscion nebulosus) y el pargo canané (Ocyurus chrysurus). En el CIAD-Unidad Mazatlán se han hecho análisis con el pargo flamenco (Álvarez-Lajonchère et al., 2011; Abdo-de la Parra et al., 2015) y el botete diana (Abdo-de la Parra et al., 2013); y de manera reciente con el robalo plateado del Pacífico, Centropomus viridis (Ibarra-Castro et al., 2017). A partir de dichos estudios se han definido modelos experimentales para el cultivo de reproductores, desove inducido, así como para la larvicultura y la 
producción de crías en pequeños contenedores bajo esquemas de alto control (recirculación) con el suministro de alimento vivo, cultivado en paralelo también a nivel de laboratorio.

A pesar del indudable valor del conocimiento generado, los alcances logrados hasta el momento son limitados y distan aun de alcanzar la escala productiva necesaria para impactar en el mercado económico.

\section{¿Cómo avanzar en la atención de este desafío?}

Desde hace décadas, China ha desarrollado modelos productivos como el sistema acoplado de desove e incubación, el cual responde con eficacia al vasto potencial biológico de distintos peces de relevancia alimentaria y comercial, incrementando la productividad en el desove, la incubación y la producción de larvas (ver figura 2).

Figura 2. Esquema del funcionamiento del sistema acoplado de desove e incubación con paso automático del huevo por vasos comunicantes (Escárcega, 2005).

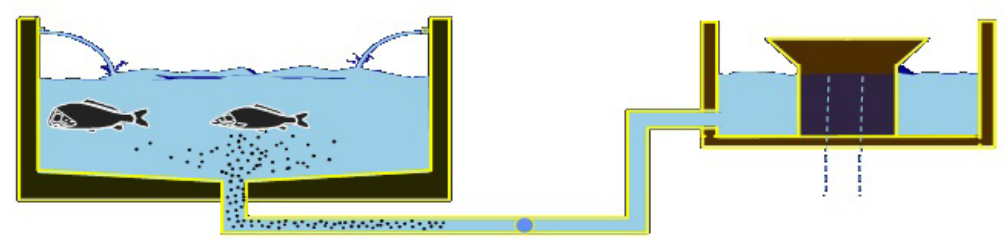

De igual manera, para la generación de crías a gran escala, se han desarrollado esquemas de cultivo larval en sistemas abiertos tales como los estanques, que permiten el manejo de grandes cantidades de larvas, con márgenes de sobrevivencia que alcanzan hasta 60\% para la etapa de larva en estadio de alimentación a cría de 3 cm (Horváth, Tamás y Coche, 1986). En el caso de los peces marinos, países como Tailandia y Australia manejan con éxito esta alternativa para la producción comercial de crías del robalo del Indo pacífico, Lates calcarifer (Kungvankij et al., 1985).

\section{Cultivo larval extensivo. El encuentro de dos rutas productivas}

El problema con la eficacia del cultivo larval extensivo estriba en coordinar la preparación de los estanques de crianza con el momento en que las larvas se encuentran en estadio de alimentación, a las 48 horas de nacidas, pues en ese momento han agotado sus reservas y se encuentran aptas para comer pequeñas presas vivas. El estanque debe tener abundancia de rotíferos y menor proporción de copépodos y otros componentes del zooplancton que en esta etapa pueden ser depredadores o competidores alimentarios. 


\section{Fundamentos del modelo}

Cuando se emplean estanques de tierra para la larvicultura, sin importar el tipo de agua, las larvas se integran desde un inicio en la cadena alimentaria que se establece en ellos. La fotosíntesis contribuye a la producción de materia vegetal en los estanques, que sirve de base para el desarrollo del zooplancton. Así, las larvas de los peces se alimentan vorazmente de él, escogiendo aquellos organismos cuya talla se ajusta a las pequeñas dimensiones de su boca (ver figura 3), como algunos rotíferos y los nauplios de copépodos.

Figura 3. Tamaños adecuados en el zooplancton (rotíferos y nauplios) en la siembra inicial de larvas de peces marinos eurihalinos en estanques (Horváth, Tamás y Coche, 1986).

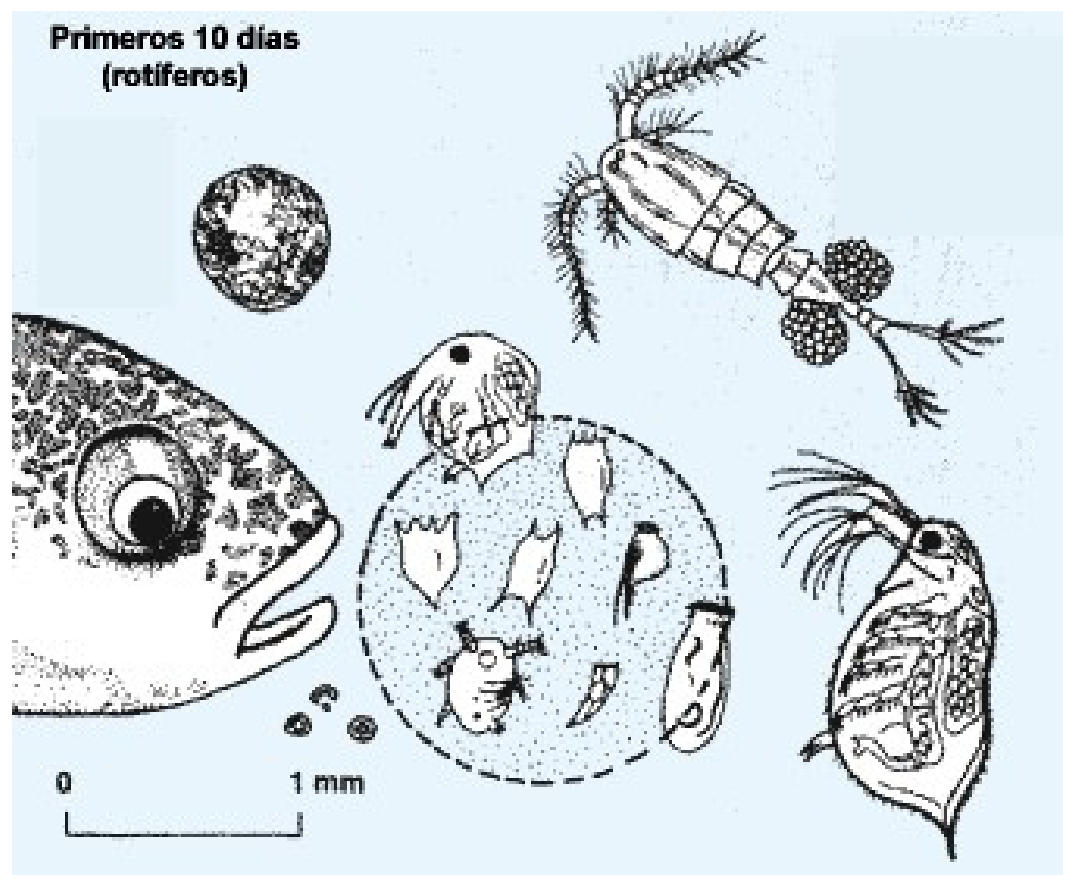

\section{Preparación de la estanquería para la recepción de las larvas}

De la misma forma en que se limpia la casa antes de que lleguen invitados, se necesita una preparación especial para alistar el ambiente para recibir a las larvas y asegurar una producción exitosa. Por supuesto, las cantidades se podrán calibrar en cada sitio conforme a los resultados que se vayan logrado en la proliferación del zooplancton. Los pasos son los siguientes:

1. Los estanques - relativamente pequeños (de 0.01 a 1.0 ha), con una profundidad media de $1.0 \mathrm{~m}$ - requieren pasar por un período de secado y exposición al sol.

2. Debe arrancarse toda la vegetación del fondo y quemarse. Luego se aplica cal viva (150 kg/ha) sobre el fondo del estanque para esterilizarlo y mejorar la estructura del suelo. 
3. Se agrega fertilizante orgánico (estiércol de ganado vacuno o porcino, por ejemplo), 3-5 ton/ha, para asegurar un buen desarrollo de zooplancton, alimento de las larvas.

4. Posteriormente, el estanque debe ser llenado a la mitad de su capacidad.

\section{Tratamiento selectivo opcional: el manejo de la sucesión natural del zooplancton}

Unavez que el estanque se hallenado a la mitad, setrata conel producto Crustabay ${ }^{1}$ (metrifonato 90\%) a una dosis de $1.0 \mathrm{mg} / \mathrm{L}$ para eliminar selectivamente a los cladóceros y copépodos², que son competidores alimentarios o depredadores de las larvas de los peces, lo que favorece la proliferación de rotíferos. Conforme van reapareciendo, los cladóceros y copépodos dejan de ser problema para los peces y se vuelven una fuente de alimento.

En las primeras pruebas se podrán sembrar las larvas en los estanques a una tasa de 740,000 unidades/ha, como se hace en el cultivo larval extensivo con la corvina ocelada del Atlántico (Sciaenops ocellatus) (FAO, 2005-2018).

\section{Acoplamiento de fases: la clave del éxito}

En la figura 4 se presenta un modelo de articulación de ambos procesos: la disponibilidad de larvas de 48 horas cuando se da la eclosión, la salida del embrión del huevo, con el momento de máxima abundancia de rotíferos. Como ha sido mencionado, basado las características de cada especie y del sitio, se deberán determinar los tiempos precisos para alcanzar los estadios señalados. Este esquema toma como ejemplo el caso del robalo, donde el punto de encuentro de las dos vías para la siembra de las larvas será en el séptimo día. Se puede apreciar que la esencia de esta ruta productiva recae en el dominio tecnológico de la reproducción de las especies objetivo, disponiendo de los lotes de larvas en estadio de alimentación de forma segura y en los momentos esperados.

Producto utilizado en México en el cultivo de camarón, para prevenir enfermedades y eliminar depredadores en los estanques de crecimiento.

${ }^{2}$ En realidad, el metrifonato actúa selectivamente sobre artrópodos en general. De esta manera, se eliminan también momentáneamente en el estanque todo tipo de crustáceos, insectos acuáticos y sus larvas, que crean problemas de mortalidad en los peces, favoreciendo una mayor supervivencia en la crianza.

\section{Conclusiones y perspectivas}

La piscicultura marina constituye una alternativa al deterioro que enfrentan distintas pesquerías en el planeta y a la necesidad creciente de impulsar opciones a fin de aumentar la oferta de alimentos y el desarrollo regional sustentable ante el crecimiento poblacional esperado a futuro. Los litorales de México, en su franja tropical, incluyen peces marinos de alto valor comercial con potencial acuícola, se destacan en este rubro los peces eurihalinos (Escárcega-Rodríguez, 2018).

En virtud de lo anterior, resulta indispensable atender uno de los aspectos sustantivos para potenciar su aprovechamiento: la producción de crías a escala 
Figura 4. Cronograma de acoplamiento de las fases para la siembra de las larvas de robalo en los estanques de crianza. Recuperado de Escárcega (2008).

\begin{tabular}{|l|l|l|l||l||l|l||l|}
\hline FASE & DÍA 1 & 2 & 3 & 4 & 5 & 6 & 7 \\
\hline $\begin{array}{l}\text { Llenado y } \\
\text { fertilización }\end{array}$ & & & & & & & \\
\hline $\begin{array}{l}\text { Reposo } \\
\text { (Floculación) }\end{array}$ & & & & & & & \\
\hline $\begin{array}{l}\text { Tramiamiento } \\
\text { selectivo }\end{array}$ & & & & & & & \\
\hline $\begin{array}{l}\text { Proliferación } \\
\text { rotiferos }\end{array}$ & & & & & & & \\
\hline $\begin{array}{l}\text { Siembra de } \\
\text { larvas }\end{array}$ & & & & & & & \\
\hline $\begin{array}{l}\text { Absorción } \\
\text { vitelo }\end{array}$ & & & & & & & \\
\hline Eclosión & & & & & & & \\
\hline Desove & & & & & & & \\
\hline $\begin{array}{l}\text { Inducción } \\
\text { hormonal }\end{array}$ & & & & & & & \\
\hline $\begin{array}{l}\text { Selección de } \\
\text { reproductores }\end{array}$ & & & & & & & \\
\hline
\end{tabular}

comercial; por lo que se considera importante aplicar el modelo de producción alternativo que aquí se presenta, pues abre la posibilidad de poder manejar enormes cantidades de larvas con mayores márgenes de sobrevivencia.

\section{Referencias}

* Abdo-de la Parra, María Isabel, Rodríguez-Ibarra, L. Estela, García-Aguilar, Noemí, Velasco-Blanco, Gabriela, \& Ibarra-Castro, Leonardo. (2013). Biotecnología para la producción masiva de juveniles del botete diana Sphoeroides annulatus: inducción hormonal y cultivo larvario. Revista de biología marina y oceanografía, 48(3), 409420. DOI: HTTPS://DX.DOI.ORG/10.4067/S0718-19572013000300001.

* Abdo-de la Parra, M. I., Rodríguez-Ibarra, L. E., Rodríguez-Montes de Oca, G., Velasco-Blanco, G. \& Ibarra-Castro L. (2015). Estado actual del cultivo de larvas del pargo flamenco (Lutjanus guttatus). Latin American Journal of Aquatic Research, 43(3): 415-423. Recuperado de: https://scielo.conicyt.cl/pdf/lajar/v43n3/art03. pdf.

* Álvarez-Lajonchère, L. S. y M. Tsuzuki Y. (2008). A review of methods for Centropomus spp. (snooks) aquaculture and recommendations for establishment of their culture in Latin America. Aquaculture Research, 39(7): 684-700.

* Álvarez-Lajonchère, L., Abdo de la Parra, M. I., Rodríguez-Ibarra, L. E. \& GarcíaOrtega, A. (2011). Reproducción controlada. En L. Álvarez-Lajonchere \& A. Puello-Cruz (Eds.), El pargo flamenco: Lutjanus guttatus, producción controlada de huevos, larvas y juveniles (pp. 25-58). Clave Editorial. Recuperado de: http:// aquaticcommons.org/14961/1/pargo flamenco libro.pdf.

* Caballero-Chávez, V. (2011). Reproducción y fecundidad del robalo blanco (Centropomus undecimalis) en el suroeste de Campeche. Ciencia Pesquera,19(1), 3546. 
* conapesca (2014). Anuario Estadístico de Pesca 2014. Comisión Nacional de Acuacultura y Pesca. México.

* Escárcega-Rodríguez, S. (1996). Evaluación de un sistema acoplado de desove e incubación para la reproducción controlada de la carpa herbívora (Ctenopharyngodon idellus). Ciencia Pesquera, 13, 87-93. Recuperado de: https:// www.inapesca.gob. $\mathrm{mx} /$ portal/documentos/publicaciones/cienciapesquera/ CP13/cp13-13.pdf.

* Escárcega-Rodríguez, S. (2005). El robalo. Avances biotecnológicos para su crianza. AGT Editor.

* Escárcega-Rodríguez, S. (2008). Guía práctica para el cultivo del robalo prieto del Pacífico Oriental Centropomus nigrescens (Günther, 1864) [documento de trabajo de la Maestría en Limnología y Acuicultura, INIRENA-UMSNH].

* Escárcega-Rodríguez, S. (2018). Preselección de especies para la piscicultura marina en el Pacífico Sur de México. CIENCIA Ergo-Sum, 25(1). Dol: https://doi. org/10.30878/ces.v25n1a6.

* Food and Agriculture Organization (FAO). (s.f.a). 10. Nutrición y alimentación de los peces. Recuperado de: http://www.fao.org/tempref/Fl/CDrom/FAO Training/ FAO Training/General/x6709s/x6709s10.htm.

* Food and Agriculture Organization (FAo). (s.f.b). 6. Fertilización de los estanques piscícolas. Recuperado de: http://www.fao.org/tempref/Fl/CDrom/FAO Training/ FAO Training/General/x6709s/x6709s06.htm.

* Food and Agriculture Organization (FaO). (2005-2018). Cultured Aquatic Species Information Programme Sciaenops ocellatus. Programa de información de especies acuáticas [texto de Cynthia K. y Faulk, A.]. Recuperado de: http://www. fao.org/fishery/culturedspecies/Sciaenops ocellatus/es.

* Food and Agriculture Organization (FAO) (2016). El estado mundial de la pesca y la acuicultura 2016. Contribución a la seguridad alimentaria y la nutrición para todos. Recuperado de: http://www.fao.org/3/a-i5555s.pdf.

* Horváth, L., Tamás, G., y Coche, A. G. (1986). La carpa común. Parte 2. Producción masiva de alevines y jaramugos. Colecc. Fao: Capacitación, 9, 83.

* Ibarra-Castro, L, Navarro-Flores, J., Sánchez-Téllez, J. L., Martínez-Brown, J. M., Ochoa-Bojórquez, L. A. and Rojo-Cebreros, Á. H. (2017). Hatchery Production of Pacific White Snook at CIAD-Unity Mazatlan, Mexico. World Aquaculture, September: 25-29. Recuperado de: https://www.researchgate.net/publication/320106950 Hatchery Production of Pacific White Snook at CIAD-Unity Mazatlan Mexico.

- Johnson, G. David. (1984). Percoidei: development and relationships. En Moser, H. G. (Ed.), Ontogeny and Systematics of Fishes (pp. 464-469). Allen Press Inc.

* Kungvankij P., Tiro, L. B. Jr, Pudadera, B. J. Jr \& Potests, I. O. (1985). Training Manual Biology and Culture of Sea Bass (Lates calcarifer). Network of Aquaculture Centres in Asia. 
* Tucker, J. W. (1987). Snook and tarpon snook culture and preliminary evaluation for commercial farming. The progressive Fish-Culturist, 49, 49-57.

\section{Cómo citar este artículo}

* Escárcega Rodríguez, Sergio (2020). ¿Cultivo de peces marinos? Hablemos de larvicultura en estanques. Revista Digital Universitaria (RDU). Vol. 21, núm. 2 marzoabril. Dol: http://doi.org/10.22201/codeic.16076079e.2020.v21n2.a3.

Recepción: 08/01/2019. Aprobación: 24/07/2019 